\title{
Performance of relay-enabled uplink in cellular networks - a flow level analysis
}

\author{
D. C. Dimitrova and G. Heijenk \\ University of Twente, The Netherlands \\ d.c.dimitrova@ewi.utwente.nl \\ geert.heijenk@utwente.nl
}

\author{
J. L. van den Berg \\ University of Twente, \\ TNO ICT, The Netherlands \\ J.L.vandenBerg@ewi.utwente.nl
}

\begin{abstract}
Uplink users in cellular networks, such as UMTS/ HSPA, located at the edge of the cell generally suffer from poor channel conditions. Deploying intermediate relay nodes is seen as a promising approach towards extending cell coverage and increasing data rates. This paper focuses on the role of packet scheduling in cellular networks with relay nodes. In particular, two uplink scheduling schemes deploying the relay functionality in different ways are compared in performance to a reference scenario where relaying is not used. We derive expressions which characterize for each of the two relay-enabled schedulers the service area of a relay station as a function of the relay location and transmit power. The results show that the service area is significantly influenced by the type of scheduling. Examining the impact on the effective data rates of mobile stations shows that there is a combination of the relay's position and transmit power which optimizes uplink service. Furthermore, based on calculations of flow throughput, we show that relaying improves flow level performance of all users, including those who do not use the relay.
\end{abstract}

\section{INTRODUCTION}

Mobile operators register a steadily increasing number of customers who expect to be connected everywhere at all times. In response, the customer driven mobile industry sets high demands toward the underlying wireless technologies to provide high data rates and wide coverage. Pervasive wireless coverage is hindered by the construction landscape of the area, e.g. buildings or other obstacles. An elegant way to improve performance in a poor coverage area is to position an intermediate relay station (RS). A relay breaks up a long direct communication path into two short indirect paths. Given a well chosen position of the RS, indirect paths can provide better channel conditions than direct paths. In addition, relaying can be incorporated on both downlink, from base station (BS) to mobile station (MS), and uplink, from MS to BS. The application of relaying for coverage improvement has been studied for a broad range of wireless technologies, including WiMAX, see [2], [15], and cellular networks, see [13], [14], [17].

In [3] we proposed a broader application of relaying, namely to improve the effective data rates of remote users. As several studies show, see [5], [8], [12], users at the edge of the cell generally suffer from strong signal degradation and thus low data rates. We see relaying as a promising approach to upgrade performance. In [3] the first step towards a more thorough analysis was made by evaluation based on data rates. The current paper extends our work from [3] by incorporating flow level analysis in the model and subsequent evaluation in terms of flow throughput. Flow level analysis is crucial since it reflects the effects of the dynamically changing number of users in a real network.

We concentrate on the EUL (Enhanced Uplink) technology for UMTS (Universal Mobile Telecommunications System), see [1]. Its high implementation percentage in Europe and North America and growing on other continents makes it a good candidate to show the benefits of relaying for operators and mobile users. However, our approach has the potential to extend towards other cellular systems such as the LTE (Long Term Evaluation) technology, which is currently under standardization.

The idea of relaying is rather attractive, however its implementation is not trivial. Placing a relay station within the cell poses multiple deployment decisions such as where to position the relay or when to use relaying. The combined effect of these decisions on MSs' performance shapes the optimal deployment solution. For example, [17] elaborates on the density of relays for the downlink in beyond-3G networks.

The type of the applied scheduling scheme is essential for the use of relays. It is important to realize that for the uplink the channel access is shared among all active users. Transmissions are organized by the base station (BS) via time frames with fixed length, 2 or $10 \mathrm{~ms}$, termed TTI (Transmission Time Interval). Depending on location, a MS's transmit capacity may be insufficient to utilize the available base station resource, in which case several scheduling strategies become an attractive choice. Some studies, for example [5], [9], [12], dedicated to scheduling in the EUL, show the relevance of the scheduler for performance.

Our goal in this paper is to evaluate the combined impact of the relay characteristics and the scheduling scheme on the performance of the mobile users. In particular, we are interested in finding the relay location and transmit power, which maximize the overall performance. Two relay-enabled scheduling schemes, which differ in the management of the relay traffic, are proposed and investigated. For each scheme the performance of the mobile stations at different locations is evaluated in terms of effective data rates and flow throughput. In addition we derive explicit analytical expressions to determine the relay's service area, which are subsequently 


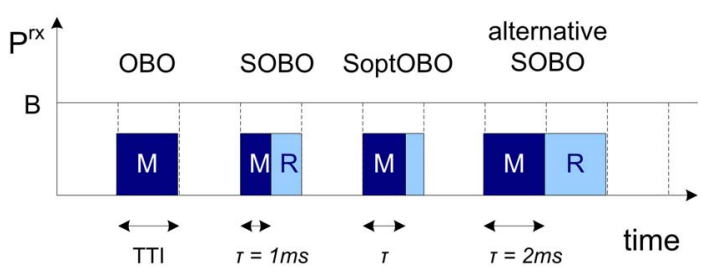

Fig. 1. Scheduling schemes: ОВO, SOBO, SoptOBO, SidOBO and ЕxOBO

supported by numerical results.

Our work is related to [11] which discusses similar relay configurations for HSDPA (High Speed Downlink Packet Access), i.e. the downlink. Although insightful, [11] does not provide any analytical derivations. On the contrary, [16] proposes several deployment scenarios with detailed discussion on the spectrum utilization but does not consider the impact of particular scheduling schemes and other system characteristics. The effect a relay has on flow level performance on an uplink we discuss in [4]. However, the considered scenario of unlimited number of relay stations is somehow unpractical. Compared to the above mentioned studies the most prominent contributions of the current paper are: analytically defining the boundaries of a service area and performance evaluation capturing the dynamic flow behaviour. Both analyses capture scheduling as well as RS specifics.

The paper continues as follows. First, in Section II, we briefly discuss the relaying concept and describe the scheduling schemes considered in this paper. The model description and analysis appear in Section III and Secion IV, respectively. Section V presents our findings of the performance evaluation. Finally, Section VI summarizes our work.

\section{RElays And SCHEduling Schemes}

First, we discuss how relaying can be applied in a cellular system and introduce relevant notation. Next, the scheduling schemes considered in this paper are described.

Relaying artificially breaks up a long communication path into two shorter ones leading to decreased path loss and increased received powers. These gains are partly lost to increased transmission time from the additional forwarding of data at the relay node. The choice between a direct path (MS-BS) and an indirect path (MS-RS-BS) is best determined based on the data rates realized on both paths. In a relayenabled system the indirect path consists of two sub-paths, MS-RS and RS-BS, and thus the effective data rate depends on the data rates realized on the two sub-paths.

Each (sub-)path is characterized by a set of transmission parameters: the distance between the communicating devices $d_{z z}$, the path loss $L_{z z}$, the transmit power $P_{z z}^{t x}$, the duration of a transmission opportunity $\tau_{z z}$ and the instantaneous data rate $r_{z z}$ during a transmission opportunity. The index $z z$ refers to the specific (sub-)path, i.e. $m s$ for the direct path from MS to $\mathrm{BS}, m r$ for the sub-path from MS to RS, and $r s$ for the sub-path from RS to BS. The transmission times $\tau_{m r}$ and $\tau_{r s}$ are scheduler specific; their sum is denoted by $\tau=\tau_{m r}+\tau_{r s}$.

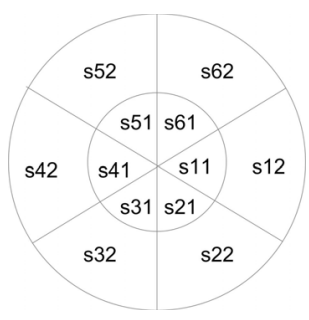

Fig. 2. Division of the cell into $S \times K$ segments, where $s$ is the number of equal-sized sectors and $K$ the number of equal sized zones. In the example $K=2$ and $S=6$.

Any further relations between the transmission parameters are discussed in Section IV. We now continue with introducing the scheduling schemes considered in our study.

\section{Scheduling Schemes}

The proposed schedulers belong to the Round Robin (RR) family where mobile users are served one-by-one (OBO), independently of their channel conditions. The design of the schemes is based on the assumption that a RS use the same frequency band for receiving and transmitting and that the switch between modes is instantaneous.

According to several studies, for example [10], [12], OBO is rather inefficient in resource utilization if users with limited power capacity are served. Still we choose OBO since we expect relaying to increase the capability of a MS to fully use the available resource. All scheduling decisions are taken by the base station while the relay only retransmits the data from the mobile. The base station assigns transmit powers such that the total available resource, equal for both RS and BS and termed budget $B$, will not be exceeded.

In our study we consider two variants of a relay-aware OBO scheduler: $S O B O$ and SoptOBO. For them, depending on location, i.e. relative distance to $\mathrm{BS}$ and $\mathrm{RS}$, a MS can select the direct or the relay path. In addition, plain $O B O$ is considered as a reference scheme for the situations without a RS, i.e. a MS always transmits directly to the base station independently of its position in the cell. All schemes assign a single TTI for the transmissions on both direct and indirect paths, i.e. $\tau=2 m s$.

On the indirect path, a shared $O B O$ (SOBO) scheme divides the TTI into two equal intervals of $1 \mathrm{~ms}$ and MS and RS both receive one interval to transmit, i.e. $\tau_{m r}=\tau_{r s}=1 \mathrm{~ms}$. The benefits of working with fixed-length transmission times for direct and indirect paths exhibit during implementation. However, static subdivision of the TTI for indirect paths is not the most efficient choice when the instantaneous rates on the sub-paths differ.

Apart from SOBO, various other schemes which apply fixed-length transmission times can be defined. For example, using a single TTI for the transmissions on both sub-paths, i.e. $\tau_{m r}=\tau_{r s}=2 m s$, meaning the BS reserves 2 TTIs for the service of a single MS. It can be shown that all schemes which, independently of precise values, use equal division of $\tau$, i.e. $\tau_{m r}=\tau_{r s}$ have the same effective data rates. Therefore, we can 
claim that SOBO is representative for a group of schedulers.

In the Optimized SOBO (SoptOBO) the channel utilization for indirect paths is optimized by adapting the transmission times on the sub-paths such that both sub-paths MS-RS and RS-BS can transfer the same amount of data, i.e. $\tau_{m r} r_{m r}=$ $\tau_{r s} r_{r s}$. Despite its maximized resource utilization SoptOBO is rather challenging for implementation since individually selecting transmission times for the sub-paths requires complex functionality in the base station.

\section{MODEL}

The model we consider consists of a single cell divided into $S$ equal-sized sectors (characterized by angle) and $K$ equalsized zones (characterized by distance), see Figure 2 . The resulting $K \times S$ segments, i.e. posible MS locations, have the same area. The number of MSs and their distribution over the segments define the system state $\underline{n}=n_{1} \cdots n_{K x S}$. With $K$ and $S$ sufficiently big we can assume that all MSs belonging to the same segment behave identically. Flow arrivals are uniformly distributed in the cell with arrival rate $\lambda$ and exponentially distributed mean flow size $F$. Since we consider the behaviour of mobile users the arrival process can be modelled correctly by a Poisson process.

A MS selects a direct or indirect path depending on which one provides higher effective data rate. All mobile stations are assumed to have the same maximum transmit power capacity $P_{m s, \text { max }}^{t x}$. The maximum transmit power of the RS is denoted $P_{r s, \max }^{t x}$. Depending on location and the available budget $B$, MS/RS use either the maximum transmit power or a lower power, i.e. $P_{z z}^{t x} \leq P_{z z \text {,max }}^{t x}{ }^{1}$. The actually applied transmit power is chosen such that a MS maximizes its utilization of the budget $B$.

At both BS and RS limited channel resource, budget $B$, is assumed. Given an operator specific noise rise $\eta$ and constant thermal noise $N$, the shared budget $B$ is derived: $B=\eta N$. Intentionally disregarding important factors such as inter- and intra-cell interference allows us to identify the effect relaying has on performance. However, we realize that such factors may have significant effect on the performance gains.

Given that we want to calculate the service area of a relay, we consider a cell with a single relay station. The service area is the aggregation of all locations within the cell from which a MS receives better service via the relay station. In addition, we are interested in how the relay position and transmit power influence MSs' performance.

\section{ANALYSIS}

In this section we concentrate on two distinctive aspects of relaying - the service area of the relay station and the impact of the relay on MS performance. In particular, we derive generic expressions which allow us to derive the service area as a function of the RS's position and transmit power.

Relaying has two opposite effects on performance. On the one hand higher received powers are enabled (increasing

\footnotetext{
${ }^{1}$ Note that $P_{m s, \max }^{t x}=P_{m r, \max }^{t x}$.
}

the effective data rate), but on the other hand forwarding at the RS requires an additional transmission of the data (decreasing the effective data rates). For each scheduler we start with calculating the received powers at the BS from which subsequently the data rates can be derived.

\section{A. Received Powers}

According to signal propagation law, the received power $P_{z z}^{r x}$ on any communication path is determined by the applied transmit power $P_{z z}^{t x} \leq P_{z z, \max }^{t x}$ and the path loss $L_{z z}\left(d_{z z}\right)$. The applied transmit power $P_{z z}^{t x}$ is determined such that its corresponding received power $P_{z z}^{r x}$ does not exceed the budget $B$. Thus we can write:

$$
P_{z z}^{r x}=\min \left(\frac{P_{z z, \max }^{t x}}{L_{z z}\left(d_{z z}\right)}, B\right)
$$

where the index $z z=(\mathrm{ms}, \mathrm{mr}, \mathrm{rs})$ denotes the (sub-)path over which the transmission is done. The assumed path loss model is given by $L(d)=123.2+10 \alpha \log 10(d)$ (in $\mathrm{dB}$ ), see [7] with $\alpha=3.52$ the path loss exponent and $d$ the distance in kilometer. The particular values of the path exponent are taken to reflect a propagation in urban model.

On the indirect path in SOBO we chose to work with the same received power at the base and relay station being determined by the slower sub-path. In SoptOBO however, according to its specification, the received powers at the base and relay station are different, as this unbalance is compensated for by difference in the transmission times, see Section II.

\section{B. Effective Data Rates}

The data rate achievable on a (sub-)path $z z$, given a particular transmit power capacity, is the instantaneous rate $r_{z z}$. Its dependency on other transmission parameters is given by:

$$
r_{z z}=\frac{r_{c h i p}}{E_{b} / N_{0}} \cdot \frac{P_{z z}^{r x}}{N+(1-\omega) P_{z z}^{r x}}
$$

In Equation (2) the index $z z=(m s, m r, r s)$ refers to the (sub-) path. $r_{c h i p}$ is the system chip rate and $E_{b} / N_{0}$ is the energy-perbit to noise ratio. We have limited the interference elements to thermal noise $N$ and self-interference accounted for by the parameter $\omega$. The maximum instantaneous rate is realized given that a single MS can fill up the budget $B$ on its own, i.e. $P_{z z}^{r x}=B$.

The effective rate $r_{\text {eff }}$ is the rate realized by a MS during its transmission opportunity with duration $\tau$. On the direct path, indexed $m s$, the effective rate is the same as the instantaneous, i.e. $r_{e f f}=r_{m s}$, because the whole transmission period $\tau$ is used by the MS. On the indirect path however, due to data forwarding, the effective rate is lower than the instantaneous. $r_{e f f}$ depends on the part of $\tau$ used by the mobile, i.e. on $\tau_{m r}$, and on the instantaneous rate that the MS can realize, i.e. $r_{m r}$. In SOBO $\tau_{m r}=\tau / 2$ while in SoptOBO $0<\tau_{m r}<\tau$ depending on MS location such that $r_{m r} \tau_{m r}=r_{r s} \tau_{r s}$ and $\tau_{m r}+\tau_{r s}=\tau$. 


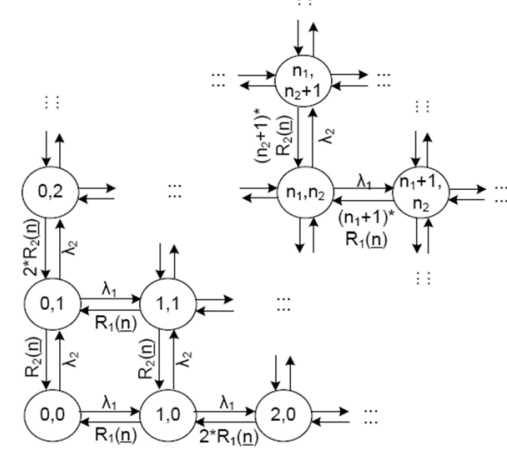

Fig. 3. Example of a two-dimensional Markov model corresponding to cell division into two segments.

Given a scheduler specific time assignment policy, the effective rate can be expressed as:

$$
r_{e f f}= \begin{cases}r_{m s} & \text { direct path in all schemes } \\ \min \left(r_{m r}, r_{r s}\right) * \frac{1}{2} & \text { indirect path in SOBO } \\ \frac{r_{m r} \tau_{m r}}{\tau} & \text { indirect path in SoptOBO }\end{cases}
$$

\section{Flow Dynamics}

The effective rate accounts for the time aspect of scheduling but fails to incorporate the effect of flow dynamics, i.e. the changing number of active users as time progresses. Since we have adopted a one-by-one scheduling strategy, a mobile station is generally not served every TTI but might have to wait for the other MSs to be served. As a result the actual data rate is lower than the effective rate. This new rate we term statedependent throughput $R_{i}(\underline{n})$. The state-dependent throughput can be derived from the MSs specific effective rate $r_{e f f, i}$ by $R_{i}(\underline{n})=r_{e f f, i} / n$, where $i$ indicates in which segment the MS is active. The dependency on the system state is captured by $n=\sum_{i=1}^{K x S} n_{i}$ and $n_{i}$ is the number of MSs in the segment $i$.

We used CTMCs (Continuous Time Markov Chains) to model the flow behaviour of the cell. The transmission rates are taken from the segment arrival rates $\lambda_{i}=\lambda /(K \times S)$ and the state-dependent throughput $R_{i}(\underline{n})$. Due to the division of the cell in $K \times S$ segments the resulting Markov model also has $K \times S$ dimensions. A simple example of a two dimensional model corresponding to cell division in only two segments is presented in Figure 3.

The form of the resulting CTMSs corresponds to the form of the CTMS of a multi-class $\mathrm{M} / \mathrm{M} / 1$ processor sharing (PS) queuing model. (The departure rates depend on how many other users share the access to the base station.) The steady state distribution of M/M/1//PS is readily available, e.g. [6]. The details of the Markov chain are scheduler specific via $R_{i}(\underline{n})$. After finding the steady-state distribution we can derive performance measures such as mean flow transfer times:

$$
D_{i}=\frac{F}{r_{e f f, i} *(1-\rho)}
$$

where $\rho$ is the system load being the sum of the loads in each segment, i.e. $\rho=\sum_{i=1}^{K \times S} \rho_{i}$, where $\rho_{i}=\lambda_{i} * F / r_{e f f, i}$ as

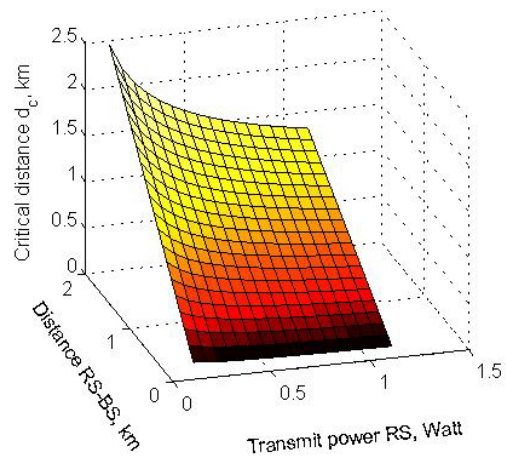

Fig. 4. Graphical representation of the dependency of the critical distance $d_{c}$ on the Rs characteristics - location and transmit power.

$i=1, \cdots, K \times S$ indicates the segment number; $F$ is the mean file size.

Hence, the average flow throughput $T_{i}$, defined as $F / D_{i}$, is given by:

$$
T_{i}=r_{e f f, i} *(1-\rho)
$$

\section{Service area of a relay station}

Our goal in this sub-section is to analytically determine the service area of a relay station, i.e. the area where a MS prefers the indirect path via the relay over the direct path. In a previous research, i.e. [3], we discussed in detail the service area based on received powers. We showed that the shape and surface of the service area depend on whether received powers or effective data rates are used in the analysis. When based on received powers the service area suggests considerable performance gain if relaying is used. However, due to data forwarding the gains in effective rates are lower than what received powers 'promise'. Therefore, in the present paper we base the calculations on effective rates.

In order to keep calculations tractable, yet providing useful insights, we consider the scenario when BS, RS and MS lay on a straight line. Both schemes - SOBO and SoptOBO are discussed as we provide analytical expressions to find the critical distance. We begin with discussion on SOBO.

1) Shared OBO: A MS always selects the path, i.e. direct or indirect, which provides higher effective data rate. The critical distance $d_{c}$ is then the distance $d_{m s}$ for which direct and indirect paths realize the same effective rate. According to Equation (3), the condition can be formally expressed as:

$$
r_{m s}=\min \left(r_{m r}, r_{r s}\right) * \frac{1}{2}
$$

Note that, given a straight line BS-RS-MS, when $d_{m r}>d_{r s}$ holds indirect transmission is chosen by default. Substituting with Equations (1) and (2) and solving for $d_{m s}$, we can derive a formal expression for $d_{c}$, namely:

$$
d_{c}=d_{r s} *\left(2 \frac{P_{m s, \max }^{t x}}{P_{r s, \max }^{t x}}\right)^{1 / \alpha}
$$


This general expression takes both characteristics of the relay - transmit power $P_{r s}^{t x}$ and position $d_{r s}$ - as parameters; $\alpha$ is the path loss exponent. The dependency is graphically presented in Figure 4. When the RS has the same transmit capacity as a mobile the equations reduces to $d_{c}=d_{r s} *(2)^{1 / \alpha}$.

2) Optimized SOBO: The discussion continues with analysis for SoptOBO. From Equation (3) follows that the critical distance $d_{c}$ is determined by the equality:

$$
r_{m s}=\frac{r_{m r} \tau_{m r}}{\tau}
$$

Per definition SoptOBO is optimized for the transfer of equal amount of data on both sub-paths. Considering the limitations set on the transfer times, we can express $\tau_{m r}$ in terms of rates, namely:

$$
\begin{aligned}
r_{m r} \tau_{m r} & =r_{r s} \tau_{r s} \\
\tau_{m r}+\tau_{r s} & =\tau
\end{aligned} \Rightarrow \tau_{m r}=\tau * \frac{r_{r s}}{r_{m r}+r_{r s}}
$$

After several substitutions and solving Equation (8) for $d_{m s}$, the critical distance can be given as a function of the positions of both, the relay and the mobile station. For a solution dependable only on relay characteristics we need to solve the system of equations:

$$
\begin{aligned}
d_{c} & =\left(d_{m r}^{\alpha}+\frac{P_{m s}^{t x}}{P_{r s}^{t x}} \cdot d_{r s}^{\alpha}\right)^{1 / \alpha} \\
d_{c} & =d_{m r}+d_{r s}
\end{aligned}
$$

In general it is difficult to solve (10) explicitly and we therefore use numerical approaches. Again if we assume that the relay has the same transmit capacity as a mobile station the first equation in (10) simplifies to $d_{c}=\left(d_{m r}^{\alpha}+d_{r s}^{\alpha}\right)^{1 / \alpha}$.

\section{NUMERICAL RESULTS ON RELAYING}

This section presents a quantitative evaluation of the impact a relay station has on the performance of mobiles. All results are analytically generated applying expressions (3) and (5) for each segment of the model of Section III. The discussion of the results starts in Sections V-B with the evaluation of the influence of key relay characteristics such as position and transmit power on effective data rates. Since these parameters are relay and not scheduler related we present the results only for SOBO.

Subsequently, the service area of a relay station is investigated in Section V-C. For each segment of the cell model we compare for both SOBO and SoptOBO whether direct or indirect path provides higher effective rate.

Finally, the three scheduling schemes - OBO, SOBO and SoptOBO - are compared in terms of effective data rates and flow throughputs. Using spatial graphs we show how relaying can improve performance and what the differences between the relay-enabled scheduling schemes are.

\section{A. Parameter Settings}

In the numerical experiments we apply a system chip rate $r_{\text {chip }}$ of $3840 \mathrm{kchips} / \mathrm{s}$, thermal noise level $N$ of $-105.66 \mathrm{dBm}$ and noise rise target $\eta$ of $6 \mathrm{~dB}$. From the given noise rise and

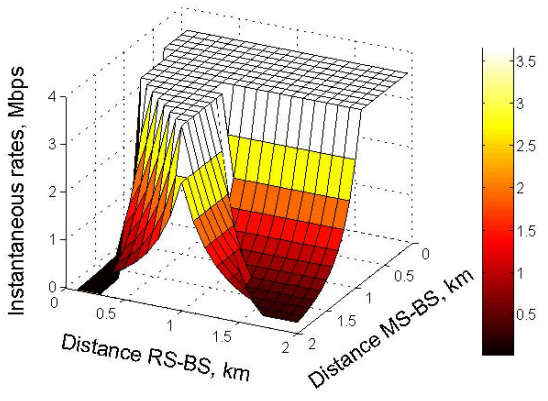

(a) Position of RS - Instantaneous rates

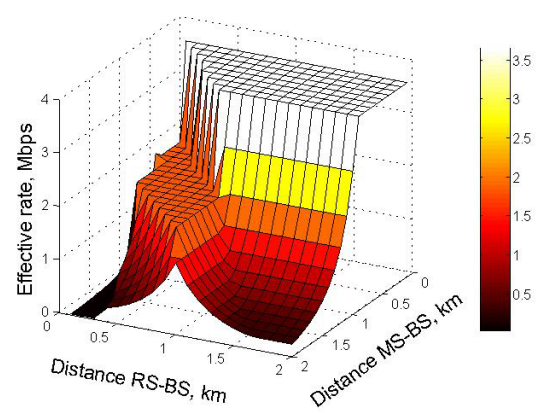

(b) Position of RS - Effective rates

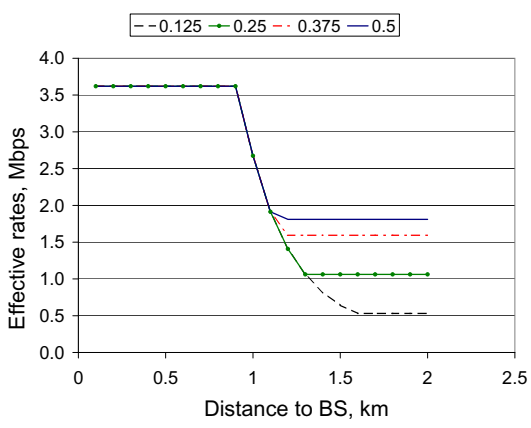

(c) Tranmsit power of RS, $d_{r s}=1.3 \mathrm{~km}$

Fig. 5. Impact of RS characteristics on MS performance with SOBO scheduler: (a) RS position on the instantaneous rates; (b) RS position on the effective rates; (c) RS transmit power.

thermal noise the available budget at both BS and RS can be calculated: $B=\eta N$. Self-interference of $10 \%$ is considered, i.e. $\omega=0.9$ in (2). All EUL flows are taken with $E_{b} / N_{0}$ target of $5 \mathrm{~dB}$. The cell range is $2 \mathrm{~km}$. As a default value a single relay station is located at $1 \mathrm{~km}$ from the base station and has $P_{r s}^{t x} 0.125$ Watt. Mobile stations have maximum transmit power $P_{\max }^{t x}=0.125$ Watt. Flows arrive with rate $0.6 \mathrm{MSs} / \mathrm{sec}$ and mean flow size of $1 \mathrm{Mbit}$.

\section{B. Characteristics of the Relay Station}

1) Position: The three dimensional graph in Figure 5(a) illustrates the impact of the location of both mobile and relay station on the instantaneous data rates. It appears that a RS at about $1 \mathrm{~km}$ delivers optimal overall performance - largest number of mobile stations with maximized effective rates. Moving the relay closer to the BS causes poor performance 
for remote MSs due to increased signal degradation. Moving the relay further away towards the edge of the cell has a twofold effect. On the one hand, increased distance to the base station leads to lower RS effective rates. On the other hand, fewer MSs can benefit from relaying and thus transmit via the direct path potentially limiting their own transmission.

The complex effect relaying has on performance is well illustrated by comparing Figures 5(a) and 5(b). Figure 5(b) presents the dependency of the effective rate on the location of both mobile and relay station. Mobile stations which are capable to fully utilize the total BS resource - on their own or supported by the relay - maximize their instantaneous rate, i.e. flat area in Figure 5(a). In terms of effective rate however such MSs show distinctive behaviour. On the direct path the effective rate is the same as the instantaneous, i.e. higher flat area in Figure 5(b). On the indirect path, due to data forwarding by the relay, it is twice smaller, i.e. lower flat area in Figure 5(b). The performance of MSs who cannot fill up the budget $B$ depends on their position and degrades as the distance increases.

2) Transmit power: Figure 5(c) presents the dependency of the effective rates on the transmit power of the relay. The performance on the straight line BS-RS-MS is observed for RS transmit powers $(0.1250 .250 .3750 .5)$ Watt. The RS is located at $1.3 \mathrm{~km}$ from the base station. Given a cell radius of $2 \mathrm{~km}$, all indirect transmissions are relay-limited, which explains the lower flat line segment in Figure 5(c). When $P_{r s}^{t x}$ reaches 0.5 Watt the relay can fully utilize the budget $B$. Further increase in the transmit power will lead to no improvement since the transmissions are now limited by the available resource $B$.

When MSs transmit directly to the base station the graphs coincide as differences start to appear after the relay is used. Interestingly, the higher the RS transmit power the earlier indirect transmissions become beneficial, i.e. the begin point of the lower flat lines moves to the left. By increasing the transmit power we enable the relay to realize higher BS budget utilization, i.e. higher data rates, than a MS located in front of the relay can achieve.

The maximum effective rate $r_{e f f, \max }$ of a MS is realized when the budget $B$ can be fully utilized. On the direct path $r_{e f f, \max }$ of $3.6 \mathrm{Mbps}$ can be achieved at distances up to $0.9 \mathrm{~km}$ according to Equations (1) and (2) respectively. Due to data forwarding, $r_{e f f, \max }$ on the indirect path is twice smaller, i.e. $1.8 \mathrm{Mbps}$ for $P_{r s}^{t x}=0.5$ Watt.

\section{Service Area}

In this section the service area of a relay station at $1 \mathrm{~km}$ from the base station and with transmit power of 0.125 Watt is discussed. The service area is based on effective data rates. The results for SOBO and SoptOBO are presented in Figures 6(a) and 6 (b) respectively. The light surface corresponds to the service area, i.e. indirect transmissions, and the dark surface to all locations from which direct transmissions are preferred.

Comparing Figures 6(a) and 6(b) we can conclude that using SoptOBO extends the area mainly for locations far from the relay while the changes for close ones are negligible. The

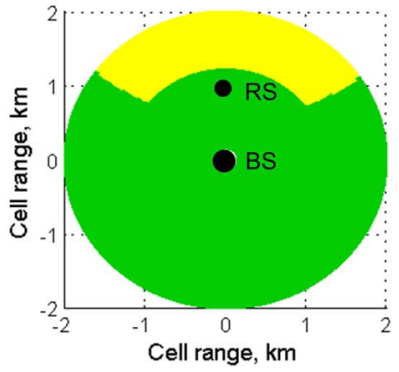

(a) Service area $\mathrm{SOBO}$

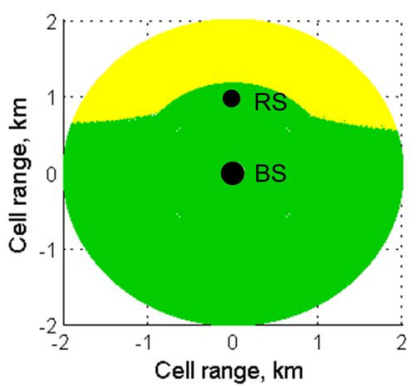

(b) Service area SoptOBO
Fig. 6. Service area of (a) SOBO and (b) SoptOBO with a relay station located at $1 \mathrm{~km}$ and default transmit power 0.125 Watt.

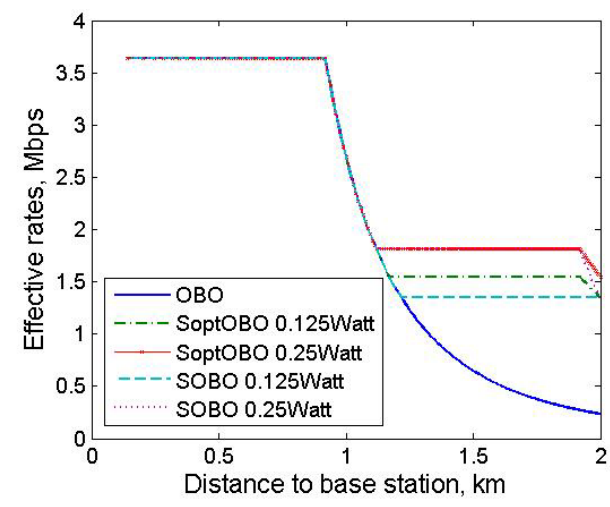

Fig. 7. Effective data rates for $\mathrm{OBO}, \mathrm{SOBO}$ and SoptOBO for two different values of the relay transmit power -0.125 and 0.25 Watt. The line BS-RS-MS is presented.

bigger the difference in effective rates on the two sub-paths for SOBO, the bigger the improvements SoptOBO can offer. Since this difference is larger for remote self-limiting MSs they also gain the most with SoptOBO.

Note that the service area does not start right behind the relay which supports our claim in Section IV-D that the actual service area is smaller than the improvements in received powers may suggest. Solving Equations (7) for SOBO on the straight line BS-RS-MS results in critical distance $d_{c}=1.22 \mathrm{~km}$. Concurringly, in Figure 6(a) the service area behind the relay start at approximately the same distance.

Knowing the service area of a relay could assist us in choosing the number of relays to cover the whole outer part of the cell.

\section{Effective Data Rates}

The distribution of the effective rates over the cell area for the three schedulers - OBO, SOBO and SoptOBO is shown in Figures 8(a) to 8(c). The brightness changes from high to low as the rates decrease. MSs located in the central white sector around the BS with radius $0.9 \mathrm{~km}$ can fully utilize the available budget $B$. In all scenarios where relaying is used performance visibly improves, e.g. twice or more higher effective rates. Furthermore, a relay configuration with SoptOBO scheme delivers better performance and larger 


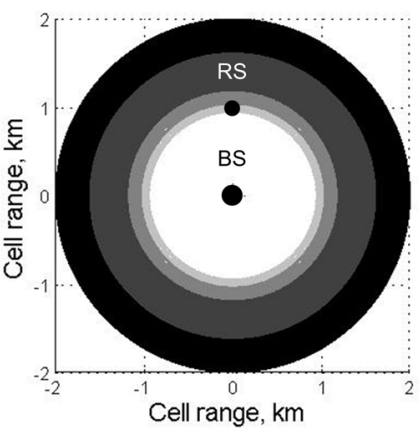

(a) Effective rate $\mathrm{OBO}$

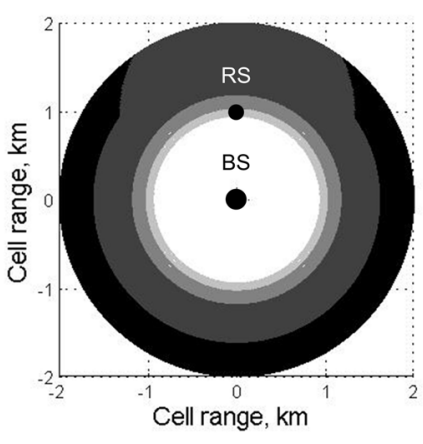

(b) Effective rate SOBO

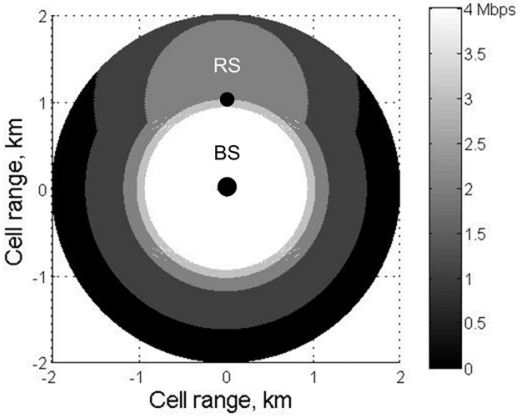

(c) Effective rate SoptOBO

Fig. 8. Effective data rates in Mbps realized with (a) OBO; (b) SOBO; and (c) SoptOBO given a relay station located at $1 \mathrm{~km}$ and default transmit power 0.125 Watt.

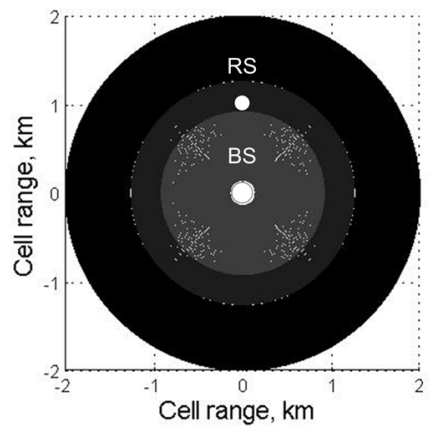

(a) Flow throughput OBO

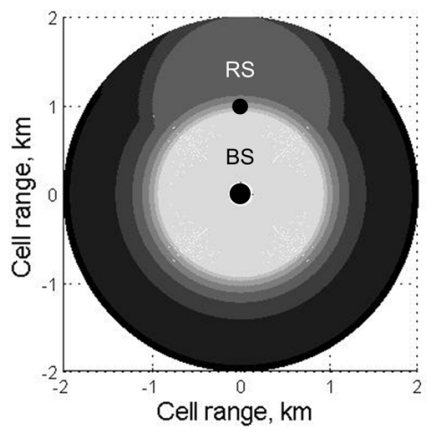

(b) Flow throughput SOBO

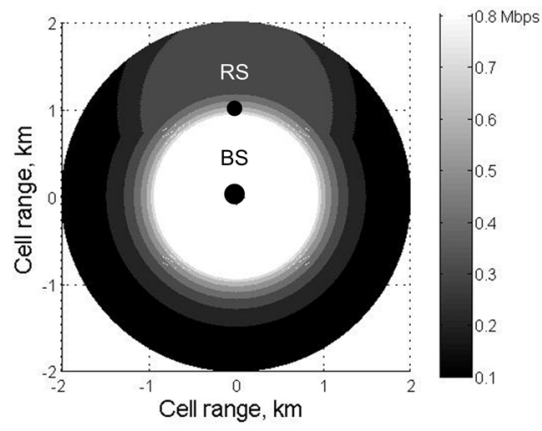

(c) Flow throughput SoptOBO

Fig. 9. Mean flow throughput in Mbps realized with (a) OBO; (b) SOBO; and (c) SoptOBO given a relay station at $1 \mathrm{~km}$ from the base station and transmit power 0.125 Watt.

service area than a SOBO scheduler offers. The strategy of SoptOBO to adaptively select the times $\tau_{m r}$ according to the MS location gives each mobile the opportunity to optimize its effective rate. The improvement degree depends, as explained, on the difference in effective data rates on the two sub-paths.

The effect of doubling the RS transmit power on the effective rates is presented in Figure 7. Increasing the RS transmit power is only beneficial for RS-limited transmissions, i.e. MSs close to the relay, and only if the RS cannot fill up the EUL budget, i.e. $P_{r s}^{r x}<B^{\prime}$. Therefore the improvements for SOBO are significant. However, in SoptOBO the highly optimized budget utilization does not leave a lot of room for improvement, see Figure 7.

\section{E. Flow throughput}

On the flow level, the benefits of relaying expand towards MSs which use the direct path. As Figure 9 illustrates, introducing a single relay station already leads to significant improvements in the mean flow throughput of all MSs irrespectively of their location. Note that the color intensity indicates the realized value as black corresponds to the lowest and white to the highest. MSs close to the base station or at the cell edge but far from the relay also register better performance, i.e. brighter color, even if they do not transmit via the RS. The particular improvements of both throughput and coverage depend on the scheduling scheme - as Figure 9 shows the SoptOBO scheme outperforms the SOBO.

The more detailed Figures 10(a) and 10(b) provide better quantitative evaluation based on the mean flow throughput realized on the straight line MS-RS-BS (Figure 10(a)) and its opposite line, i.e. at 180-degrees (Figure 10(b)). Selecting these two opposite directions allows us to show that the impact of the relay station is applicable for the whole cell. The graphs suggest that relaying can improve flow performance up to 5 times for close MSs and up to 20 times for remote MSs which use the relay, see Figure 10(a). Furthermore, as we observed for the effective rates, increasing the transmit power of the relay could lead to further improvements.

The overall improvement at the flow level can be explained by considering the system (cell) occupancy. Increased effective rates for remote users translate into shorter service time and decreased system load, which influence positively all MSs, see Equation (5). The poor performance of the OBO scheme is the result of inefficient resource utilization and thus long service time. As Figure 10(b) shows remote MSs which use direct path register the smallest improvement due to low effective rates.

We argue in Section I that a representative performance evaluation should consider flow dynamics. As Figures 10(a) and $10(\mathrm{~b})$ indicate the analysis delivers crucial performance 


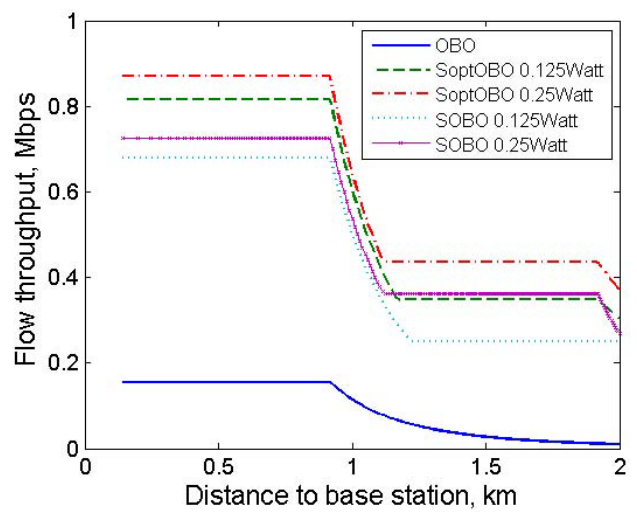

(a) Line BS-RS-MS

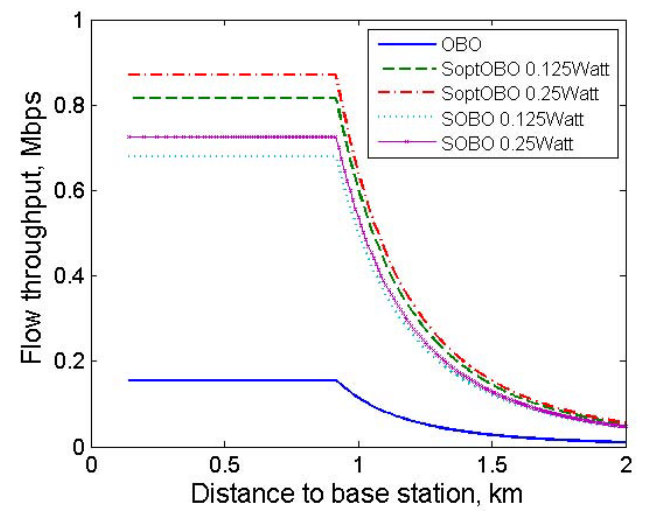

(b) Line BS-MS

Fig. 10. Mean flow throughput with a relay station located at $1 \mathrm{~km}$ and transmit power 0.125 Watt. (a) corresponds to the straigh line BS-RS-MS and (b) to the line in the opposite direction.

insights and allows us to observe the long-term effects of relaying. We can conclude that accounting for the flow behaviour of MSs is a viable part of the system evaluation.

\section{CONCLUSIONS}

This paper discussed the benefits of relaying on the uplink by comparing the performance of two relay-enabled schemes to a reference scheduler with no relay. We provided an analytical approach to describe the service area of a relay station based on effective data rates. The analysis is supported by simulations. The results show that the shape and size of the service area depend on the relay characteristics but also on the scheduling schemes. We also evaluated how relay characteristics, such as transmit power and location, affect the effective rates of the mobiles. Specifically, we have determined the optimal position of the RS which optimizes the overall performance. Particular, valuable contribution of the current paper is the flow level analysis (taking into account that the number of active users fluctuates over time), which showed that relaying has a long-term positive effect on the all mobile users independently whether they use the relay or not. Furthermore, we established that the maximum possible data rate is mostly limited not by the relay or the mobile but by the available resource at the base station.

A topic for further research is considering the impact of environment parameters such as inter-cell interference. We believe that application of relay stations will have significant, positive influence on the interference generated by the cell due to the lower applied transmit powers. Our intention is to examine this issue along with the performance of a relayenabled scheme in the presence of inter-cell interference.

Acknowledgments We are grateful to Remco Litjens from TNO ICT, The Netherlands for the appropriate remarks on modeling and for his constant enthusiasm and readiness to help.

\section{REFERENCES}

[1] 3GPP TS 25.309. FDD Enhanced Uplink; Overall Description.

[2] Albert Bel and Gonzalo Seco-Granados Jose Lopez Vicario. The benefits of relay selection in WiMAX networks. In ICT-MobileSummit' $08,2008$.

[3] D. C. Dimitrova, H. van den Berg, and G. Heijenk. Analyzing the impact of relay station characteristics on uplink performance in cellular networks. ERCIM eMobility proceedings '09, Enschede, The Netherlands, 2009.

[4] D. C. Dimitrova, H. van den Berg, and G. Heijenk. Performance analysis of uplink packet schedulers in cellular networks with relaying. WMNC '09, Gdansk, Poland, 2009.

[5] D. C. Dimitrova, H. van den Berg, G. Heijenk, and R. Litjens. Flow-level Performance Comparison of Packet Scheduling Schemes for UMTS EUL. volume 5031. WWIC '08, Tampere, Finland, 2008.

[6] B. Haverkort. Performance of computer communication systems. John Wiley \& Sons Ltd, 1999.

[7] H. Holma and A. Toskala. HSDPA/HSUPA for UMTS. John Wiley \& Sons Ltd, 2006.

[8] You Jin Kang, Junsu Kim, Dan Keun Sung, and Seunghyun Lee. Hybrid scheduling algorithm for guaranteeing qos of real-time traffic in high speed uplink packet access (hsupa). pages 1-5, 2007.

[9] A. Mäder, D. Staehle, T. Liu, and H. Barth. Feasible load regions for different RRM strategies for the enhanced uplink in UMTS networks. In Springer, Lecture Notes in Computer Science, volume 4396, pages 213-228. EuroNGI Workshop, 2006.

[10] S. Ramakrishna and J. M. Holtzman. A scheme for throughput maximization in a dual-class CDMA system. ICUPC '97, San Diego, USA, 1997.

[11] Eike Reetz, Rainer Hockmann, and Ralf Tonjes. Performance Study on Cooperative Relaying Topologies in Beyond 3G Systems. In ICTMobileSummit '08, 2008.

[12] C. Rosa, J. Outes, T.B. Sorensen, J. Wigard, and P.E. Mogensen. Combined time and code division scheduling for enhanced uplink packet access in WCDMA. IEEE VTC '04 (Fall), Los Angeles, USA, 2004.

[13] R. Schoenen, R. Halfmann, and B.H. Walke. An FDD Multihop Cellular Network for 3GPP-LTE. pages 1990-1994, 2008.

[14] Martina Umlauft. Relay Devices in UMTS Networks - effects on Application Performance. In Proceedings of the Fifth Annual Mediterranean Ad Hoc Networking Workshop (Med-Hoc-Net 2006), 2006.

[15] Josep Vidal, Olga Munoz, Adrian Agustin, Eduard Calvo, and Andreu Alcon. Enhancing 802.16 networks through infrastructure-based relays. In ICT-MobileSummit '08, 2008.

[16] E. Weiss, S. Max, O. Klein, G. Hiertz, and B. Walke. Relay-based vs. Conventional Wireless Networks: Capacity and Spectrum efficiency. pages 1-5. Personal, Indoor and Mobile Radio Communications, 2007. PIMRC 2007. IEEE 18th International Symposium on, 2007.

[17] Marc Wener, Peter Moberg, and Per Skillermark. Cost assessment of radio access network deployment with relay nodes. ICT-MobileSummit 2008 Conference Proceedings, 2008. 